\title{
Su una speciale classe di serie di funzioni analitiche.
}

\author{
Memoria $2^{a}$ di Lutar ONorri (a Bologna).
}

Sunto. - L'A. studia le funzioni analitiche definite da serie della forma

$$
\sum_{n=0}^{\infty} a_{n z} z^{n} f_{n}\left[\varphi^{n} n(z)\right]
$$

essendo $\mathrm{f}_{\mathrm{n}}(\mathrm{u}), \varphi(\mathrm{z})$ frnzioni intere assegnate.

In un lavoro recentemente pubblicato in questi "Annali » (1), ho esposto un metodo generale per determinare i campi di convergenza uniforme delle serie del tipo

\section{essendo:}

$$
\sum_{n=0}^{\infty} \alpha_{n} z^{n} f_{n}\left[\varphi^{p_{n}}(z)\right]
$$

i coefficienti $\alpha_{n}$ delle costanti;

gli esponenti $p$, dei numeri interi, positivi tendenti all'infinito;

le funzioni $f_{n}(u), \varphi(z)$ analitiche, intere e la $\varphi(z)$ non costante.

Nella presente Memoria mi occupo della rappresentazione di una funzione analitica assegnata $F(z)$ mediante serie del tipo (1) e della ricerca dei punti singolari di $F(z)$.

Prima di riassumere brevemente il contenuto di questo lavoro, credo opportuno di ricortare al lettore quei risultati esposti nella prima Memoria ai quali dovro ricorrere frequentemente nel seguito.

Indicati con $M_{n}\left(r^{p_{n}}\right)$ il massimo di $f_{n}(u)$ sulla circonferenza $\left(0, r^{\hat{w}_{n}}\right) \mathrm{e}$ con $R, R(r)$ i raggi di convergenza delle serie di potenze

$$
\sum_{n=0}^{\infty} a_{n} z^{n}, \sum_{n=0}^{\infty} \alpha_{n} M_{n}\left(r^{n} p_{n}\right) z^{n}
$$

ho dimostrato che il campo $C$ formato con i punti $z$ del piano nei quali è

$$
R(|\varphi(z)|+0)-|z|>0
$$

(1) L. Onofri, Su una speciale classe di serie di funeioni analitiche. "Annali di Matematica pura ed applicata s, Tomo XII, nn. 1-2, 1983-34. 
gode delle seguenti proprietà :

a) In ogni campo limitato e completamente interno a $C$ la serie (1) converge uniformemente.

b) La (1) non può convergere uniformemente nell'intorno di un punto esterno a $C$.

Se poi si suppone $f_{n}(0)=1$ e la successione delle $f_{n}(u)$ egualmente limitata in un intorno della origine, si prova facilmente che il campo $C$ è contenuto nel cerchio $(0, R)$.

Nella prima parte di questa Memoria, considero una serie di potenze di $z$, avente raggio di convergenza $\delta>0$, e mi propongo di determinare una serie del tipo (1) che abbia la proprieta di convergere uniformemente in ua intorno della origine e di rappresentare nel medesimo intorno la funzione analitica $F(z)$ definita dalla serie di potenze considerata.

Supposto $\varphi(0)=0, f_{n}(0)=1$ ed indicato con $r^{\prime}$ il limite superiore dei valori di $r$ pei quali la successione

$$
M_{0}\left(r^{p_{0}}\right), M_{1}\left(r^{p_{1}}\right), \ldots, M_{n}\left(r^{p}{ }^{p}\right), \ldots
$$

è limitata superiormente e con $\left(0, \rho^{\prime}\right)$ la circonferenza su cui il massimo di $\mid \varphi(z)$ : è eguale a $r^{\prime}$, è sempre possibile determinare i coefficienti $a_{*}$ in modo che la corrispondente serie (1) rappresenti la $F(z)$ almeno nel minore dei due cerchi $(0, \delta),\left(0, \rho^{\prime}\right)$.

Se risulta $\rho^{\prime} \geq \delta$, il campo $C$ contiene tutto il cerchio $(0, \delta)$ e, talrolta, anche punti esterni a questo cerchio. Beneinteso, se cio accade, il contorno di $C$ deve passare pei punti singolari della $F(z)$ posti sulla circonferenza $(0, \delta)$.

La seconda parte della Memoria è dedicata appunto allo studio dei casi in cui $C$ è più ampio di $(0, \delta)$ ed alla ricerca dei punti singolari della $F(\approx)$ posti sulla periferia di questo cerchio. In particolare, si trova che se $\rho^{\prime}=\delta$, $R \neq \delta$ e se la $\varphi(z)$ non è della forma $k z^{n / k}(k$ costante, $m$ intero, positivo), i punti singolari di $F(z)$, aventi modulo $\delta$, sono in numero finito e soddisfano l'equazione $|\varphi(z)|=r^{\prime}$.

È noto che sulla circonferenza di convergenza di una serie di potenze esiste almeno un punto singolare della funzione analitica definita dalla serie stessa. Un fatto analogo non sempre si riscontra nelle serie da me considerate in quanto può avvenire che le funzioni $F(z)$, definite da esse, siano regolari nell' interno e sul contorno di $C$.

Nella terza parte di questo lavoro ho esaminata la questione stabilendo un gruppo di proposizioni che, in numerosi casi, sono sufficienti a rivelare il comportamento di $F(z)$ sal contorno di $C$. 
Una di queste proposizioni può riguardarsi come l' estensione alle serie (1) di un noto teorema sulle serie di potenze dovuto al VIVANT.

I.

1. Consideriamo una successione di numeri interi positivi

$$
p_{0}, p_{1}, \ldots, p_{n}, \ldots
$$

tendenti all'infinito ed una successione di funzioni analitiche intere

$$
f_{0}(u), f_{1}(u), \ldots, f_{n}(u), \ldots
$$

eguali all' unità nella origine.

Posto, per comodità di scrittura,

$$
f_{n}(u)=1+u g_{n}(u)
$$

indichiamo con $G_{n}\left(r^{p}{ }^{p}\right)$ il massimo della funzione $\left|g_{n}(u)\right|$ sulla circonferenza $\left(0, r^{p} n\right)$ e con $r^{\prime}$ il limite superiore dei valori di $r$ pei quali la successione

è limitata superiormente.

$$
r^{p_{n}} G_{n}\left(r^{p_{n}}\right) \quad(n=0,1, \ldots)
$$

E facile dimostrare che per ogni $r<r^{\prime}$ è:

$$
\lim _{n \rightarrow \infty} r_{n} G_{n}\left(r_{n}^{x_{n}}\right)=0
$$

Difatti, preso un numero $r_{1}$ soddisfacente alle disuguaglianze

$$
r<r_{1}<r^{\prime}
$$

e determinato un numero positivo $A$ in modo che si abbia:

$$
r_{1} p_{n} G_{n}\left(r_{1}{ }^{p_{n}}\right)<A
$$$$
(n=0,1, \ldots)
$$

si può scrivere, in forza delle (4) e (5),

$$
r_{n}^{p_{n}} G_{n}\left(r_{n n} p_{n} \leq\left(\frac{r}{r_{1}}\right)^{p_{n}}{ }_{r_{1}} p_{n} G_{n}\left(r_{1}{ }^{n}{ }_{n}\right)<\left(\frac{r}{r_{1}}\right)^{p_{n}} A\right.
$$

2. Nelle questioni che tratteremo più avanti, il numero $r^{\prime}$, dianzi definito, verrà, di norma, supposto positivo. In generale, tale numero non può superare l'unità richiedendo l'ipotesi opposta un particolarissimo comportamento delle funzioni $f_{n}(u)$. Ed invero, si ha che:

Se $\mathrm{r}^{\prime}>1$, le funzioni $\mathrm{f}_{\mathrm{n}}(\mathrm{n})$ tendono uniformemente all' unitò in ogni regione finita del piano.

Evidentemente, basterà provare la tendenza uniforme allo zero delle funzioni $u g_{n}(u)$ in un cerchio $(0, S)$ di raggio $S$ scelto comunque. 
All' nopo, si fissino ad arbitrio un $r>1$, ma inferiore ad $r^{\prime}$, ed un $\varepsilon>0$. In virtù della (3) e della tendenza all'infinito degli esponenti $p_{n}$, sarà possibile determinare un indice $\bar{n}$ in modo che per $n>\bar{n}$ si abbia, ad un tempo,

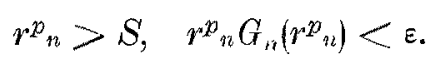

Ciò vuol dire che, per $n>\bar{n}$ e $|u| \leq S$, è:

$$
\left|u g_{n}(u)\right|<\varepsilon \text {. }
$$

3. Sia $E(z)$ una funzione analitica definita da una serie di potenze

$$
F(z)=\sum_{n=0}^{\infty} A_{r_{i}} z^{n}
$$

avente il raggio di convergenza $\delta$ maggiore di zero.

Vogliamo vedere se si può determinare una serie del tipo (1), ma differente dalla (6), la quale sia atta a rappresentare, in un conveniente intorno della origine, la funzione data $F(z)$.

Per precisare maggiormente i dati della questione, ammetteremo che le funzioni $f_{n}(u)$ siano del tipo (2) e che la $\varphi(z)$ sia nulla per $z=0$.

Cominciamo intanto con l'osservare che:

Una serie del tipo (1), convergente uniformemente in un intorno della origine, rappresenta la $\mathrm{F}(\mathrm{z})$ nel medesimo intorno, se e soltanto se $i$ coefficienti $\mathrm{a}_{\mathfrak{n}}$ soddisfano alle relazioni

$$
\begin{aligned}
& A_{0}=F(0)=a_{0} . \\
& A_{n}=\frac{F^{(n)}(0)}{n !}=\sum_{\nu=0}^{n-1} \frac{a_{\nu}}{(n-v) !} D^{n-\nu} \varphi^{p_{\nu}}(0) g\left[\varphi^{p_{\nu}}(0)\right]+a_{n} \quad(n=1,2, \ldots) .
\end{aligned}
$$

Posto:

$$
F_{1}(z)=\sum_{y=0}^{\infty} a_{y} z^{y} f_{v}\left[\varphi^{p}(z)\right]
$$

si dividano ambo i membri di questa eguaglianza per $z^{n+1}(n=0,1, \ldots)$ e si integrino poi, così divisi, lungo una circonferenza $(0, \rho)$ contenuta nell' intorno in cui l' eguaglianza stessa è valida. Eseguendo, al $2^{\circ}$ membro, l'integrazione termine a termine ed approfittando del teorema integrale di CAUCHY, si perviene ad una eguaglianza del tipo (7), salvo il cambiamento di $F^{(n)}(0)$ in $F_{1}^{(m)}(0)$.

Quindi, se $F(z)=F_{1}(z)$, valgono le $(7)$, e viceversa se queste relazioni sono soddisfatte, si ha, per ogni $n, F^{(n)}(0)=F_{1}^{(n)}(0)$ e ciod $F(z)=F_{1}(z)$.

È utile notare come le (7) ci assicurino l'unicita della soluzione del problema, in corrispondenza di un prefissato sistema di funzioni $f_{n}(u), \varphi(z)$ e di numeri $p_{n}$. 
4. La proposizione precedente ei permette, in sostanza, di ricondurre la questione enunciata al n. 3 alla ricerca del campo $O$ di convergenza uniforme di una serie del tipo (1) i cui coefficienti $a_{n}$ soddisfano alle (7). E per giungere alla conoscenza di $O$ ci serviremo dei metodi e dei risultati che trovansi esposti nella citata Memoria $1^{\text {a }}$.

Prendiamo una circonferenza con centro l'origine e di raggio $\rho$ qualsivoglia ed indichiamo con $r$ il massimo che la funzione $|\varphi(z)|$ assume sulla predetta circonferenza. Applicando una nota formula di CAUCHX, abbiamo:

da cui :

$$
\frac{1}{(n-v) !} D^{n-\varphi p_{\nu}}(0) g_{\nu}\left[\varphi^{p_{\nu}}(0)\right]=\frac{1}{2 \pi i} \int_{(\rho)} \frac{\varphi^{p_{\nu}}(z) g_{\nu}\left[\varphi^{x_{\nu}}(z)\right]}{z^{n+1-\nu}} d z
$$

$$
\left|\frac{1}{(n-\nu) !} D^{n-\nu} \varphi^{p_{\nu}}(0) g_{\nu}\left[\varphi^{p_{\nu}}(0)\right]\right| \leq \frac{r^{p_{\nu}} G_{\nu}\left(r^{p_{\nu}}\right)}{\rho^{n-\nu}} \quad\left(\begin{array}{l}
\nu=0,1, \ldots \\
n \geq \nu
\end{array}\right) .
$$

E poichè :

$$
\left|a_{n}\right| \leq\left|A_{n}\right|+\sum_{\nu=0}^{n-1} \frac{\left|a_{y}\right|}{(n-v) !}\left|D^{n-v} \varphi^{p_{v}}(0) g_{v}\left[\varphi^{p_{v}}(0)\right]\right|
$$

sarà anche, in forza delle (8),

$$
\left|a_{n}\right| \leq\left|A_{n}\right|+\frac{1}{\rho^{n}} \sum_{\nu=0}^{n-1}\left|a_{\nu}\right| \rho^{\nu} r^{p_{\nu}} G_{\nu}\left(r^{p_{\nu}}\right) \quad(n=1,2, \ldots) .
$$

Da questa disuguaglianza discende la seguente

$$
\left|a_{n z}\right| \leq \frac{1}{\rho^{n}} \prod_{\nu=0}^{n-1}\left[1+r^{p_{\nu}} G_{\nu}\left(r^{x_{\nu}}\right)\right] \cdot \sum_{\mu=0}^{n}\left|A_{\mu}\right| \rho^{\mu} \quad(n=1,2, \ldots)
$$

come ora mostreremo applicando il principio d'induzione.

Ritenendo valida la (10) sino all' indice $n-1$ (per $n=1$ si può verificare direttamente), si ha, per la (9),

$$
\begin{aligned}
& \left|a_{n}\right| \leq \frac{1}{\rho^{n}} \int_{\nu=1}^{n-1}\left[r^{p_{y}} G_{y}\left(r^{p_{\nu}}\right) \cdot \prod_{m=0}^{\nu-1}\left(1+r^{p_{m}} G_{m i}\left(r^{p_{m}}\right)\right) \cdot \sum_{\mu=0}^{\nu}\left|A_{k}\right| \rho^{p}\right]+ \\
& +\left|A_{0}\right| r^{p_{0}} G_{0}\left(r^{p_{0}}\right)+\left|A_{n}\right| \rho^{n} \mid \leq \\
& \leq \frac{1}{\rho^{n}}\left\{\sum _ { \nu = 1 } ^ { n - 1 } \left[r^{p_{\nu}} G_{v}\left(r^{p_{v}}\right) \cdot \prod_{m=0}^{\nu-1}\left(1+r^{p_{m}} G_{m i}\left(r^{\left.p_{m}\right)}\right)\right]+\right.\right. \\
& \left.+r^{p_{v}} G_{0}\left(r^{p_{0}}\right)+1\right\} \sum_{\mu=0}^{n}\left|A_{\mu}\right| F^{\beta}= \\
& =\frac{1}{\rho^{n}} \prod_{\nu=0}^{n-1}\left[1+r^{p_{\nu}} G_{\nu}\left(r^{p_{\nu}}\right)\right] \cdot \sum_{\mu=0}^{n}\left|A_{\mu}\right| \rho^{\mu} .
\end{aligned}
$$


Supponiamo ora che il numero $\rho$ sia, ad un tempo, inferiore a $\delta$ ed al raggio $\rho^{\prime}$ di quella circonferenza sulla quale la funzione $\mid \varphi(z)$ | assume come massimo assoluto il valore $r^{r}$ definito al n. ${ }^{\circ}$. Allora, preso ad arbitrio un $\varepsilon>0$, si potrà determinare, in forza della (3), un indice $\bar{v}$ in guisa che per $\vee>\bar{v}$ sia:

$$
r^{p_{\nu}} G_{\nu}\left(r^{p_{\nu}}\right)<\varepsilon
$$

Tenendo conto di cio, dalla (10) si ricava:

$$
\left|a_{\nu}\right|<\frac{1}{\rho^{n}} \prod_{\nu=0}^{\bar{\nu}}\left[1+r^{p_{\nu}} G_{\nu}\left(r^{p_{\nu}}\right)\right] \cdot(1+\varepsilon)^{n-\bar{\nu}-1} \cdot \sum_{\mu=0}^{n}\left|A_{\mu \nu}\right| \rho^{\mu},
$$

da cui, per l'arbitrarietà di $\varepsilon$ e per essere $\rho<\delta$,

$$
\varlimsup \sqrt[n]{\left|a_{n}\right|} \leq \frac{1}{\rho}
$$

Questa disuguaglianza serve ad assicurarci che il raggio di convergenza $R$ della serie

$$
\sum_{n=0}^{\infty} a_{n} z^{n}
$$

non può essere inferiore ad entrambi i numeri ò e $\rho^{\prime}$.

Passiamo, infine, ad esaminare la funzione $R(r)$ ('). Poichè:

$$
\left|a_{n}\right| M_{n}\left(r^{p_{n}}\right) \geq\left|a_{n}\right|
$$

si avrà : $R(r) \leq R$, e poichè, per $r<r^{\prime}$ e per $n$ sufficientemente elevato, è:

$$
\left|a_{n}\right| M_{n}\left(r^{p_{n}}\right) \leq\left|a_{n}\right|\left[1+r^{x_{n}} G_{n}\left(r^{p_{n}}\right)\right] \leq\left|a_{n}\right|(1+\varepsilon),
$$

si avrà anche: $R(r) \geq R$.

Onde:

$$
R(r)=R \quad \text { per } \quad r<r^{\prime}
$$

Da tutto ciò consegue il seguente

Teorema. - Se:

a) la serie di potenze

$$
F(z)=\sum_{n=0}^{\infty} A_{n} z^{n}
$$

ha il raggio di convergenza o maggiore di zero;

(1) Per il significato delle notazioni qui usate, vedi il $n^{\circ} 2$ della Memoria $1^{a}$ e l'intro. duzione del presente lavoro. 
b) le funzioni $\mathrm{f}_{\mathrm{n}}(\mathrm{u})$ sono del tipo (2) e la $\varphi(\mathrm{z})$ è nulla nell' origine;

c) il numero $\mathrm{r}^{\prime}$ è positivo;

d) $i$ coefficienti $\mathrm{a}_{\mathrm{n}}$ soddisfano alle (7);

allora:

il numero $\mathrm{R}$ non è inferiore ad entrambi $i$ numeri $\rho^{\prime}$ e $\delta$;

il campo $\mathrm{C}$ di convergenza uniforme della $(1)$ è contenuto nel cerchio $(0, \mathrm{R})$ e contiene tutti $i$ punti $\mathrm{z} d i$ questo cerchio nei quali $\grave{e}|\varphi(\mathrm{z})|<\mathrm{r}^{\prime}$;

in ogni regione connessa contenente l'origine ed appartenente a $\mathrm{O}$ la serie (1) rappresenta la funzione data $\mathrm{F}(\mathrm{z})$; in particolare, tale rappresentazione è valida nel cerchio comune $a\left(0, \rho^{\prime}\right)$ e $(0, \delta)$.

5. Esempio. - Proponiamoci di rappresentare la funzione $F(z)$, individuata dalla (6), mediante una serie del tipo

$$
F(z)=A_{0}+\sum_{n=1}^{\infty} a_{n} z^{n}\left(1+\frac{z^{n}}{k_{n}}\right)
$$

essendo $i$ numeri $k_{n}$ tutti differenti dallo zero e tali che la suecessione $\sqrt[n]{\left|k_{n}\right|}$ abbia limite positivo per $n$ tendente all' infinito.

Si ha, evidentemente, $\rho^{\prime}=r^{\prime}=\lim _{n \rightarrow \infty} \sqrt[n]{\left|k_{n}\right|}$.

Applicando le (7) otteniamo le relazioni

$$
A_{2 n+1}=a_{2 n+1}, \quad A_{2 n}=a_{2 n}+\frac{a_{n}}{k_{n}},
$$

le quali ci forniscono i valori dei coefficienti $a_{n}$ d'indice dispari e ci permettono di determinare facilmente i coefficienti restanti. Invero, indicato con $d$ un numero dispari qualsivoglia, si scrivano le seconde delle (13) per i valori $d, 2 d, \ldots, 2^{-1} d$ dell' indice $n$ e si risolva il sistema di equazioni, così ottenuto, rispetto alle incognite $a_{2 d}, a_{d d}, \ldots, a_{2^{1}{ }^{2}}$. Operando in tal guisa, si perviene alla formola

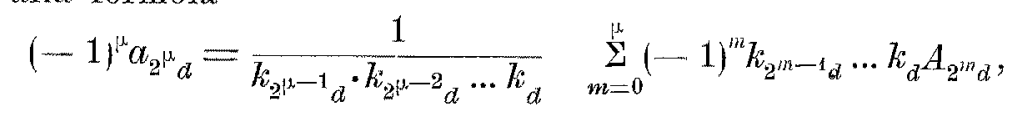

atta al calcolo dei coefficienti $a_{n}$ d'indice pari.

Premesso ciò, è facile determinare il campo $C$ di convergenza uniforme della serie (12) $\left(^{1}\right)$

(1) Alla conoscenza di $C$ si può giungere, in modo assai rapido, determinando $\mathrm{j}$ cerchi di convergenza delle serie $\Sigma a_{n} z^{n}, \Sigma \frac{a_{n} z^{2 n}}{k_{\eta n}}$ nelle quali può immaginarsi decomposta la (12), Qui pero, dato il carattere illustrativo dell' esempio, abbiamo preferito applicare i procedi. menti generali esposti al $\mathrm{n}^{0} 4$. 
Si osservi, intanto, che, per essere $\varphi(z)=z$, questo campo ì un cerchio col centro nell'origine ed il cui raggio $S$ non puó, per ragioni ovvie, saperare il raggio di convergenza $\delta$ della $(6)$.

Se $\rho^{\prime} \geq \delta$, la serie (12) converge uniformemente in tutto $(0, \delta)$, onde $\grave{e} S=\delta$.

Se invece $p^{\prime}<\delta$, il raggio $S$ assume il valore $\delta$ oppure il valore $\rho^{\prime}$ secondo che $R$ è maggiore od eguale a $\rho^{\prime}$. Difatti, per essere:

$$
R(r)=\frac{R \rho^{\prime}}{r} \quad\left(r>\rho^{\prime}\right),
$$

il contorno del campo $O$ è costituito dai punti $z$ nei quali è:

$$
\frac{R \rho^{\prime}}{|z|}=|z|
$$

e cioè $S=\sqrt{R \rho^{\prime}}$. Se poi si aresse, ad un tempo, $R>\rho^{\prime}, S<\delta$, le due serie di potenze

$$
\sum_{n=0}^{\infty}\left(A_{n}-a_{n}\right) z^{n}, \sum_{n=1}^{\infty} \frac{a_{n} z^{2 n}}{k_{n}},
$$

(identiche in forza della (12)) avrebbero per raggi di convergenza $\mathrm{i}$ due numeri distinti $\delta$ ed $S$.

Il calcolo del numero $R$ può effettuarsi agevolmente mediante le (13) e (14) che servono ad esprimere le $a_{n}$ in funzione delle quantità note $A_{n}$; ̀े però interessante osservare come in generale, e cioè salvo casi assai particolari, si trovi essere $R=\rho^{\prime}$.

Si ha, invero,

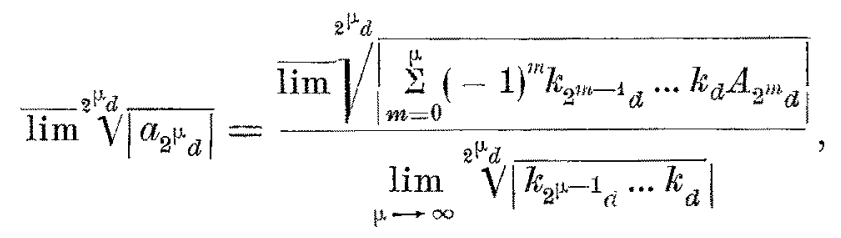

$$
\begin{aligned}
& \lim _{\mu \rightarrow \infty} \sqrt[2]{\mu_{\mu} d} \sqrt{\left|k_{2^{\mu-1} a} \ldots k_{d}\right|}=\rho^{\prime}
\end{aligned}
$$

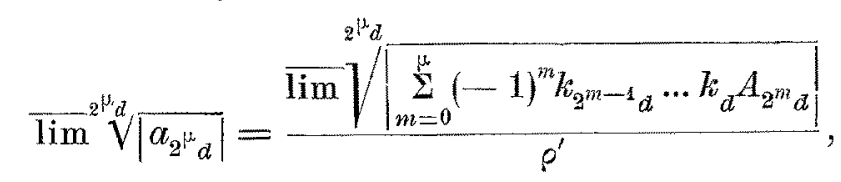

e quindi, se $R$ è maggiore di $\rho^{\prime}$, il limite massimo che figura al $2^{\circ}$ membro deve essere inferiore all' unità. 
Manifestamente, ciò richiede che sia:

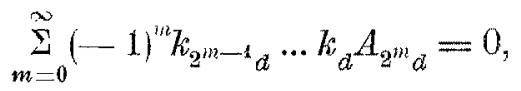

comunque si scelga il numero dispari $d$.

In generale, è dunque $R=\rho^{\prime}$ e, conseguentemente, $S=\rho^{\prime}$.

\section{II.}

6. Questo paragrafo è dedicato allo studio delle principali proprietà del campo $C$ di convergenza uniforme di una serie del tipo (1) rappresentante la funzione data $F(z)$ ed alla ricerca di criteri che servano ad assicurarci la convergenza della (1) in una regione più ampia di $(0, \delta) 0$, almeno, in tutto questo cerchio.

Ammettiamo, dapprima, che sia $\rho^{\prime}>\delta$.

In base alla (11) deve essere $R \geq \delta$; d'altra parte, se fosse $R>\delta$, la serie (1) convergerebbe uniformemente in un cerchio di raggio maggiore di $\delta$, la qualcosa è assurda possedendo la $F(z)$ almeno un punto singolare di modulo $\delta$. Quindi :

Se $\rho^{\prime}>\delta$, il campo $\mathrm{C}$ coincide con il cerchio $(0, \delta)$.

7. Passiamo a studiare il caso in cui è $\rho^{\prime}=\delta$.

Sempre dalla (11) risulta $R \geq \delta$. Se $R=\delta$, il campo $C$ coincide con il cerchio $(0, \delta)$. Se $R>\delta$, deve essere $R\left(r^{\prime}+0\right) \leq \delta$. Invero, nel caso opposto, il campo $C$ conterrebbe tutti i punti della circonferenza $(0, \delta)$; e ciò non è possibile essendovi su tale circonferenza qualche punto singolare della $F(z)$. Pertanto:

Il campo o è formato da tutti e soli $i$ punti z del piano nei quali è:

$$
|z|<R, \quad|\varphi(z)|<r^{\prime}
$$

In particolare, se $\varphi(z)=k z^{n !}(m$ intero, positivo), sulla circonferenza $(0, \delta)$ si ha sempre $|\varphi(z)|=r^{\prime}$, onde $O$ coincide con il cerchio $(0, \delta)$.

Se invece $(z)$ non è del tipo suddetto, il campo $C$ è sicuramente più ampio del cerchio $(0, \delta)$. In tal caso, la serie (1) rappresenta la $F(z)$ in una regione più estesa di quella fornita dalla ordinaria serie di TAYLoR, appartenendo alla suddetta regione tutti quei punti $z$ della circonferenza $(0, \delta)$ nei quali ̀े $|\varphi(z)|<r^{\prime}$.

Osserviamo, infine, che la funzione $F(z)$ deve possedere, sulla circonferenna $(0, \delta)$, un numero finito di punti singolari poichè, se ciò non av- 
venisse, la funzione $|\varphi(z)|$, assumendo il valore $r^{\prime}$ in infiniti punti di $(0, \delta)$, sarebbe necessariamente della forma $k z^{m}$.

Riassumendo quanto è stato esposto nel presente numero, possiamo enunciare la seguente proposizione:

Se $\rho^{\prime}=\delta$, il campo $\mathrm{C}$ coincide con il cerchio $(0$, o) qualora sia $\mathrm{R}=\delta$ oppure $\varphi(\mathrm{z})=\mathrm{kz}^{\mathrm{m}}$. In ogni altro caso, il campo $\mathrm{C} \dot{e}$ piì ampio di $(0, \delta)$ e la funzione $\mathrm{F}(\mathrm{z})$, rappresentata dalla (1), possiede, sulla circonferenza $(0, \delta)$, un numero finito di punti singolari. Questi punti z si trovano fra le soluzioni dell' equazione

$$
|\varphi(z)|=r^{\prime}
$$

8. Esempio. - Supponiamo che la funzione $F(z)$ sia rappresentata da una serie del tipo

$$
F(z)=\sum_{n=0}^{\infty} a_{n} z^{n}\left[1+b_{n} z^{z^{n}}\left(\frac{z+c}{1+c}\right)^{p}\right]
$$

essendo $a_{n}, b_{n}, c$ numeri positivi e:

$$
R>1, \quad \overline{\lim } \sqrt[n]{a_{n} b_{n}}>1, \quad \overline{\lim }_{\sqrt[p]{n}}^{\sqrt{b_{n}}}=1
$$

Si ha, manifestamente,

$$
\varphi(z)=\frac{z(z+c)}{1+c}, \quad f_{n}(u)=1+b_{n} u, \quad r^{\prime}=1, \quad p^{\prime}=1
$$

onde $\delta \geq 1$. D'altra parte, il numero $\delta$ non può superare l'unità inquantochè la serie data $\dot{\theta}$ divergente per $z=1$. Avendosi, pertanto, $\delta=\rho^{\prime}, R>\delta$, pos. siamo asserire, applicando la proposizione del $\mathrm{n}^{\circ} 7$, che il campo $C$ è formato con i punti z nei quali è:

$$
|z|<R, \quad|z(z+c)|<1+c
$$

e che la funzione $F(z)$ possiede, sulla circonferenza $(0,1)$, l'unico punto singolare $z=1$.

9 Se $\rho^{\prime}=\delta$ e se, per $\mathbf{r}>\mathbf{r}^{\prime}$, si può poire:

$$
\overline{\lim } \sqrt[n]{M_{n}\left(r^{\left.p_{n}\right)}\right.} \leq V(r),
$$

essendo $\mathrm{V}\left(\mathrm{x}^{\prime}+0\right)=1$, allora il campo $\mathrm{C}$ coincide con il cerchio $(0, \delta)$.

Si ha:

$$
\varlimsup \sqrt[n]{\left|a_{n}\right| M_{n}\left(r^{p}\right)} \leq \varlimsup \sqrt[n]{\lim } \sqrt{\left|a_{n}\right|} V(r)
$$


cioè:

$$
\begin{gathered}
R(r) \geq \frac{R}{V(r)} \\
R\left(r^{\prime}+0\right) \geq R .
\end{gathered}
$$

Ma, per quanto è stato osservato al no 7 , deve essere:

onde:

$$
R\left(r^{\prime}+0\right) \leq \delta, \quad R \geq \delta,
$$

$$
R=\delta \text {. }
$$

10. Il precedente criterio $\dot{e}$ di facile e frequente applicazione. Ad esempio: Se:

$\rho^{\prime}=\delta$

il limite massimo $\mu$ del rapporto $\frac{\mathrm{p}_{\mathrm{n}}}{\mathrm{n}} \dot{e}$ finito;

le funzioni $\mathrm{f}_{\mathrm{n}}(\mathrm{u})$ sono del tipo

$$
f_{n}(u)=1+b_{1 n} u+b_{2 n} u^{2}+\ldots+b_{m n} u^{m}
$$

con $\mathrm{m}$ indipendente $d a \mathrm{n}$;

allora:

il campo $\mathrm{C}$ coincide con il cerchio $(0, \delta)$. numeri

Poichè per $r<r^{\prime}$ la successione $M_{n}\left(r^{p_{n}}\right)$ è limitat periormente, i

da cui:

$$
\lim _{s p_{n}} \sqrt{\left|b_{s n}\right|} \quad(s=1,2, \ldots, m)
$$

non potranno superare $\frac{1}{r^{\prime}}$. Di conseguenza, preso un numero $k>\frac{1}{r^{\prime}}$ ed un $r>r^{\prime}$, si potrà determinare un indice $\bar{n}$ in modo che per $n>\bar{n}$ sia:

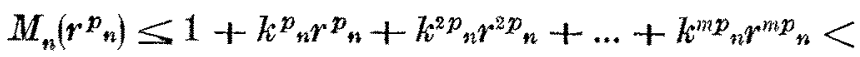

$$
\begin{aligned}
& <\frac{(k r)^{n n} p_{n}+1}{k r-1}
\end{aligned}
$$

$$
\overline{\lim } \stackrel{n}{V}_{M_{n}\left(r^{p} n\right)} \leq(k r)^{n n}
$$

ossia, per l'arbitrarietà di $k$,

$$
\overline{\lim } \sqrt[n]{\vee} \overline{M_{n}\left(r^{p} n\right)} \leq\left(\frac{r}{r^{\prime}}\right)^{m \mu}
$$

11. Esaminiamo, ora, il caso in cui si ha $\rho^{\prime}<\delta$ essendo $\delta$ un numero finito. Per ragioni di semplicita, ammetteremo che la successione $\sqrt[n]{M_{n}\left(r^{p} n\right)}$ Annali di Matematica. Sorie IV, Tomo XIIT. 
abbia limite finito per ogni valore di $r$ e porremo:

$$
V(r)=\lim _{n \rightarrow \infty} V^{n} \overline{M_{n}\left(r^{\left.p_{n}\right)}\right.}
$$

In base a tale ipotesi, si ha:

$$
R(r)=\frac{R}{V(r)}
$$

Indicati con $m$ ed $M$ il minimo ed il massimo di $|\varphi(z)|$ sulla circonferenza $(0, \delta)$, osserviamo che il limite $R(M+0)$ non può superare $\delta$, esistendo sulla predetta circonferenza qualche punto singolare della $F(z)$. Ciò richiede, per la (15), che sia:

$$
R \leq \delta V(M+0)
$$

Se $R<\delta V(M-0)$, si ha:

$$
R(r)<\delta \frac{V(M-0)}{V(r)}
$$

e quindi per valori di $r$ minori di $M$ ma abbastanza prossimi ad $M$ :

$$
R(r)<\delta \text {. }
$$

Ciò significa che il campo $O$ non paò contenere tutti i punti del cerchio $(0, \delta)$.

Supponiamo, invece, che sia $R=\delta V(M-0)$. Dalla (15) abbiamo:

ed anche:

$$
R(r)=\delta \frac{V(M-0)}{V(r)}, \quad R(r+0)=\delta \frac{V(M-0)}{V(r+0)},
$$

$$
R(r+0)>\delta
$$

purchè $r$ sia inferiore alla più piccola radice $\rho$ della equazione

$$
V(r+0)=V(M-0)\left({ }^{1}\right)
$$

Quindi, se il minimo $m$ di $|\varphi(z)|$ è inferiore a $\rho$, il eampo $C$. è più ampio di $(0, \delta)$, se, altrimenti, $m \geq \rho$, tutti i punti della circonferenza $(0, \delta)$ appartengono al contorno di $C$.

Infine, se $R>\delta V(M-0)$, dalla (15) risulta:

$$
R(r+0)>\delta \frac{V(M-0)}{V(r+0)}
$$

(1) Se non esistono xadici, al numero $\rho$ va attribuito il valore $M$. 
e, conseguentemente,

$$
R(r+0)>\delta
$$

per ogni $r<M$. Onde, il campo $C$ contiene tutti i punti della circonferenza $(0$, o) nei quali è $|\varphi(z)|<M$.

Siamo così giunti al risultato seguente:

a) Se $\mathrm{R}<\delta \mathrm{V}(\mathrm{M}-0)$, $\mathrm{C}$ non contiene tutti $i$ punti di $(0, \delta)$.

b) Se $\mathrm{R}=\delta \mathrm{V}(\mathrm{M}-0), \mathrm{m} \geq \rho$, il cerchio $(0, \delta)$ appartiene a $\mathrm{C}$ e la periferia di $(0, \delta)$ al contorno di $\mathrm{C}$.

c) Se $\mathrm{R}=\delta \mathrm{V}(\mathrm{M}-0), \mathrm{m}<\rho, \mathrm{C} \dot{e}$ pì ampio $d i(0, \delta)$ ed $i$ punti singolari della $\mathrm{F}(\mathrm{z})$, aventi modulo $\delta$, soddisfano alla disuguaglianza

$$
|\varphi(z)| \geq \rho \text {. }
$$

d) $S e \mathrm{R}>\delta \mathrm{V}(\mathrm{M}-0), \varphi(\mathrm{z})=\mathrm{kz}^{\mu}, \mathrm{C}$ coincide con $(0$, o); in ogni altro caso, $\mathrm{C}$ è più ampio di $(0, \delta)$ ed $i$ punti singolari della $\mathrm{F}(\mathrm{z})$, aventi modulo $\delta$, soddisfano alla equazione

$$
|\varphi(z)|=M
$$

12. Esempio I. - Consideriamo la serie

essendo:

$$
F(z)=\sum_{n=0}^{\infty} a_{n} z^{n}\left[1+b_{n} \varphi^{p}(z)\right]
$$

$a_{n}, b_{n}$ numeri positivi;

la serie $\sum_{n=0}^{\infty} a_{n} b_{n}$ divergente;

$\varphi^{(n)}(0)$ positiva o nulla, comunque sia $n$, e $\varphi(1)=1$;

$$
\lim _{n \rightarrow \infty} \frac{p_{n}}{n}=\mu>0, \quad \lim _{n \rightarrow \infty} \stackrel{p_{n}}{V} \overline{b_{n}}=k>1, \quad R=k^{\mu}
$$

Mediante facili calcoli, si trova essere:

$$
r^{\prime}=\frac{1}{k}, \quad \rho^{\prime}<1, \quad V(r)=(k r)^{\mu}, \quad R(r)=\frac{1}{r^{\mu}} \quad\left(r>\frac{1}{k}\right)
$$

Determiniamo, anzitutto, il numero $\delta$; poichè $R(1)=1$, il campo $C$ contiene tutti i punti del cerchio $(0,1)$, onde $\delta \geq 1$; ma non può essere $\delta>1$ inquantochè la serie data diverge per $z=1$. $\mathrm{E}$ dunque $\delta=1$.

Avendosi poi $M=1, V(M-0)=k^{\mu}=R, \rho=1$, possiamo affermare, applicando le proposizioni b) e c) del no 11 , che il campo $C$ è generalmente più ampio del cerchio $(0,1)$, coincidendo con questo cerchio soltanto se $\varphi(z)=z^{\nu}$. 
In ogni caso, i punti singolari della $F(z)$, posti sulla circonferenza $(0,1)$, soddisfano alla equazione

$$
|\varphi(\approx)|=1
$$

Esempio II. - Se $V(r)=1$, comunque sia $r$, si ha $R(r)=R, R \leq \hat{\delta}$. In conseguenza di ciò, il campo $C$ viene a coincidere con il cerchio $(0, R)$.

Questa osservazione può applicarsi nel caso in cui le $f_{n}(u)$ siano del tipo

$$
f_{n}(u)=1+b_{1 n} u+b_{2 n} u^{2}+\ldots+b_{m n} u^{m}
$$

con $m$ indipendente da $n$, ed il rapporto $\frac{p_{n}}{n}$ tenda allo zero per $n$ tendente all' infinito.

Invero, dalla disuguaglianza

$$
M_{n}\left(r^{\left.p_{n}\right)}<\frac{(k r)^{m p_{n}+1}-1}{k r-1} \quad\left(k>\frac{1}{r^{\prime}}, r>r^{\prime}\right)\right.
$$

che abbiamo dimostrata al $n^{0} 10$, si deduce:

e, per essere $M_{n}\left(r^{p_{12}}\right) \geq 1$,

$$
\overline{\lim } \sqrt[n]{M_{n}\left(r^{p}\right)} \leq 1
$$

$$
\lim _{n \rightarrow \infty} \sqrt[n]{M_{n}\left(r^{p}\right)}=1
$$

13. Vogliamo, infine, indicare un criterio di scelta per le funzioni $f_{n}(u)$, $\varphi(z)$ e per i numeri $p_{n}$ che ci assicuri la convergenza uniforme della (1) in tutto il cerchio $(0, \delta)$. In base al Teorema generale del $n^{\circ} 4$, basterà fare in modo che risulti $\rho^{\prime} \geq \delta$.

Distinguiamo due casi :

a) $\delta$ è un numero finito. Le funzioni $f_{n}(u)$ siano del tipo (2), ma del resto comnnque. Scelto un numero positivo $r_{1}<1$, si determinino gli esponenti $p_{n}$ in guisa che la successione $M_{n 3}\left(r_{1}{ }_{n}\right)$ sia limitata superiormente. E ciò è possibile avendosi $f_{n}(0)=1$.

Poi, presa una funzione intera $\biguplus(z)$ nulla nell' origine, si ponga $\varphi(z)=k \psi(z)$, avendo l'avvertenza di assumere il numero $t$ in modo che il massimo di $|\varphi(z)|$ sulla circonferenza $\left(0\right.$, o) non superi $r_{1}$.

Con una siffatta scelta, risulta certamente $\rho^{\prime} \geq \delta$.

b) $\delta$ è infinito, cioè $\mathrm{F}(\mathrm{z})$ è funzione inter $\alpha$. Converrà che sia $r^{\prime}=\infty \mathrm{e}$ quindi occorrerà che le funzioni $f_{n}(u)$ tendano uniformemente ad 1 in ogni regione finita del piano (vedi $n^{\circ} 2$ ). 
Cio ammesso, si fissino, ad arbitrio, due numeri $r_{1}, A$ maggiori di 1 ed una successione di numeri positivi $k_{\nu}$ tali che:

$$
\lim _{y \rightarrow \infty} \frac{k_{y}}{y}=\infty
$$

Si determini poi un'altra successione di numeri interi

$$
N_{1}<N_{2}<\ldots<N_{\nu}<\ldots
$$

in guisa che per $n \geq N_{\nu}$ si abbia:

$$
M_{12}\left(r_{1} k_{y}\right)<A .
$$

Questa determinazione è possibile in virtù della supposta convergenza uniforme delle $f_{s}(u)$ in ogni regione finita del piano.

Infine, si ponga:

$$
p_{n}=\nu \text { per } N_{\nu} \leq n<N_{\nu+1},
$$

lasciando arbitraria la scelta degli esponenti $p_{n}$ i cui indici $n$ risultino inferiori a $N_{1}$.

Preso allora an $r$ qualsivoglia, si ha, per ogni $v$ non inferiore ad un conveniente $\bar{v}$,

e, per $n \geq N_{\nu}^{-}$

$$
r^{y}<r_{i}^{k_{y}}
$$

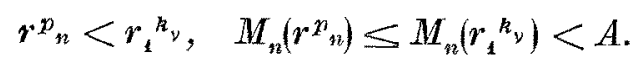

È dunque $r^{\prime}=\infty$.

Osservazione. - Questo procedimento si può applicare anche se $\delta$ è finito; anzi, esso presenta, rispetto al metodo indicato per il caso a), il vantaggio di non richiedere la conoscenza del numero $\delta$.

III.

14. A differenza di quanto avviene per le ordinarie serie di potenze, le funzioni analitiche definite da serie del tipo (1) non sempre possiedono punti singolari sul contorno $\gamma$ del campo $C$ di convergenza uniforme. In altre parole, esistono dei casi nei quali si riscontra la regolarità delle suddette funzioni nell'interno e sul contorno di $C$. Per persuadersi di ciò, basta pensare che se $R$ è inferiore a $\delta$, il campo $C$ è completamente interno al cerchio $(0, \hat{o})$.

Dalle considerazioni svolte ai $n^{1} 6,7,11$, è agevole dedurre dei criteri che ci permettano di affermare l'esistenza su $\gamma$ di punti singolari della $F(z)$. 
Così, se $\rho^{\prime} \geq \delta$ oppure se $\rho^{\prime}<\delta, R \geq \delta V(M-0)$, il contorno $\gamma$ contiene tutti i punti singolari della $F(z)$ che hanno modulo eguale a $\delta$.

15. Passiamo ora ad esporre un altro criterio che richiede soltanto un esame diretto dei coefficienti $a_{n}$ e delle funzioni $f_{n}(x)$ e $\varphi(z)$.

Poniamo:

$$
\begin{gathered}
a_{n} f_{n}(u)=\sum_{m=0}^{\infty} b_{m n} u^{m n}, \\
\varphi(z)=\sum_{m=0}^{\infty} c_{m m^{2}} z^{m}
\end{gathered}
$$

ed ammettiamo che i coefficienti $c_{m}$ siano reali non negativi e che esista un punto proprio $\delta$, distinto dalla origine, comune al contorno di $C$ ed al semiasse reale, positivo.

Sotto queste ipotesi è facile provare che: tutti i punti interni al cerchio $(0,8)$ appartengono a $\mathrm{C}$.

Invero, fissato $z$ internamente a $(0, \delta)$, determiniamo un intorno $(\delta, \rho)$ di $\delta$ in modo che nei punti $z_{1}$, appartenenti, ad un tempo, al detto intorno ed a $C$, siano verificate le disuguaglianze

$$
\left|z_{1}\right|>|z|,\left|\varphi\left(z_{1}\right)\right|>|\varphi(z)| \text {. }
$$

In eiascuno di questi punti sarả inoltre:

onde:

$$
R\left(\left|\varphi\left(z_{1}\right)\right|+0\right)-\left|z_{1}\right|>0,
$$

$$
R(|\varphi(z)|+0)-|z|>R\left(\left|\varphi\left(z_{1}\right)\right|+0\right)-\left|z_{1}\right|>0 \text {. }
$$

Pertanto, il punto $\approx$ appartiene a $C$.

È poi manifesto che il contorno di $C$ non può avere più di un punto proprio in comune con il semiasse reale, positivo.

Premesso ciò, dimostriamo il seguente

Teorema. - Se:

a) $i$ coefficienti $\mathrm{c}_{\mathrm{m}}, \mathrm{b}_{\mathrm{mn}}$ soddisfano alle disuguaglianze

$$
c_{m} \geq 0, \quad\left|\arg b_{m n}\right|<h \frac{\pi}{2} \quad(h<1)
$$

b) il contorno di $\mathrm{C}$ ed il semiasse reale, positivo hanno in comune un punto proprio $\delta$, distinto dalla origine;

allora:

la funzione $\mathrm{F}(\mathrm{z})$, definita dalla (1) nel cerchio $(0, \delta)$, è singolare per $\mathrm{z}=\delta$. 
Ammessa la regolarità della funzione $F(z)$ nel punto $\delta$, prendiamo un numero positivo $\alpha$ minore di $\delta$ ed un numero $k>\delta-\alpha$ in modo che la serie

$$
\sum_{\nu=0}^{\infty} \frac{k^{\nu}}{\nu !} F^{(\nu)}(\alpha)=\sum_{\nu=0}^{\infty} \frac{k^{\nu}}{\nu !} \sum_{n=0}^{\infty} a_{n} D^{\nu} \alpha^{n} f_{n}\left[\varphi^{p} n(\alpha)\right]
$$

risulti convergente.

Posto:

$$
A_{n \nu}+i B_{n \nu}=a_{n} D^{\nu} \alpha^{n} f_{n}\left[\varphi^{p}{ }_{n}(\alpha)\right],
$$

ed osservato che, in forza delle condizioni enunciate, è:

$$
A_{n \times} \geq\left|a_{n} D^{\nu} \alpha^{n} f_{n}\left[\rho^{p} n(\alpha)\right]\right| \eta \quad(\eta>0),
$$

separiamo, nella (16), la parte immaginaria dalla reale. Quest' ultima è rappresentata dalla serie assolutamente convergente

$$
\sum_{\nu=0}^{\infty} \sum_{n=0}^{\infty} \frac{k^{\nu}}{\nu !} A_{n \nu}
$$

Tenendo conto di ciò e della disuguaglianza

$$
\left|a_{n} z^{n} f_{n}\left[\varphi^{p}(\xi)\right]\right| \leq \sum_{\nu=0}^{\infty} \frac{|z-\alpha| \nu}{\nu !}\left|a_{n} D^{\nu} \alpha^{n} f_{n}\left[\varphi^{p} n(\alpha)\right]\right| \leq \sum_{\nu=0}^{\infty} \frac{k^{\nu}}{\nu !} \frac{A_{n v}}{\eta},
$$

valida per $|z-\alpha| \leq k$, possiamo asserire che la serie (1) converge uniformemente in un intorno del punto $\delta$. Ma questa conclusione è assurda appartenendo $\delta$ al contorno di $C$.

Dunque, la $F(z)$ non può essere regolare per $z=\delta$. 